\title{
Utilização do método hidrotérmico assistido por micro-ondas na síntese de catalisadores a base de $\mathrm{SnO}_{2}$ dopados com nióbio
}

\section{(Microwave-assisted hydrothermal synthesis of niobium-doped $\mathrm{SnO}_{2}$ catalysts)}

\author{
R. R. S. Valois ${ }^{1}$, M. M. Oliveira', J. H. G. Rangel', J. S. Vasconcelos ${ }^{3}$, C. J. S. Mendonça ${ }^{4}$, \\ A. P. Maciel $^{5}$, E. Longo ${ }^{6}$ \\ ${ }^{1} P P G E M,{ }^{2} P P G Q,{ }^{3} D E E$, IFMA , Av. Getúlio Vargas, 4, S. Luís, MA, Brasil 65025-001 \\ ${ }^{4}$ PPGEA, ${ }^{5}$ Departamento de Química, UFMA, Av. dos Portugueses, 1966, S. Luís, MA, Brasil 65080-805 \\ ${ }^{6}$ CMDMC, LIEC, Instituto de Química, UNESP, C.P. 355, Araraquara, SP, Brasil 14801-907 \\ renata.ufma@gmail.com,marcelo@ifma.edu.br,hiltonrangel@ifma.edu.br,jomar@ifma.edu.br, \\ mendoncacaritas@ig.com.br,ap.maciel@ufma.br,elson@iq.unesp.br
}

\begin{abstract}
Resumo
A síntese de nanopartículas de óxido de estanho dopado com nióbio pelo método hidrotermal assistido por micro-ondas foi investigada, utilizando como precursores o etóxido de nióbio e pentóxido de nióbio. Os catalisadores obtidos foram aplicados na reação de esterificação do ácido oleico. Através das técnicas de difração de raios X, microscopia eletrônica de varredura, espectrometria de energia dispersiva de raios $\mathrm{X}$ e espectroscopia de infravermelho com transformada de Fourier, as características estruturais e morfológicas dos pós foram analisadas. A aplicação dos catalisadores resultou em um rendimento de 41,6\% de oleato de metila. Palavras-chave: óxido de estanho, nanopartículas, esterificação, método hidrotermal assistido por micro-ondas.
\end{abstract}

\begin{abstract}
Microwave-assisted hydrothermal synthesis of niobium-doped tin oxide nanoparticles, using niobium ethoxide and niobium pentoxide as precursors, was investigated. The prepared catalysts were used in the esterification reaction of oleic acid. The structural and morphological characteristics of the powders were analyzed by X-ray diffraction, scanning electron microscopy, energy dispersive $X$-ray spectrometry, and Fourier transform infrared spectroscopy. The application of catalysts resulted in a yield of $41.6 \%$ of methyl oleate.
\end{abstract}

Keywords: tin oxide, nanoparticles, esterification, microwave-assisted hydrothermal method.

\section{INTRODUÇÃO}

O desenvolvimento de materiais nanométricos destacase como uma das áreas de maior interesse devido ao seu potencial tecnológico, visto que a necessidade de aparelhos eletrônicos menores e melhores vem crescendo exponencialmente nos últimos anos. Tais pesquisas estão presentes em diversas áreas da ciência e tecnologia, como no desenvolvimento de pigmentos [1], sensores de gases, baterias recarregáveis [2] e nas ciências biológicas [3, 4]. Dentre os materiais estudados, o dióxido de estanho $\left(\mathrm{SnO}_{2}\right)$ vem se destacando. $\mathrm{O} \mathrm{SnO}_{2}$ é um semicondutor extrínseco do tipo $\mathrm{n}$ que possui estrutura tetragonal compacta do tipo rutilo. É um óxido anfótero, ou seja, possui características ácidas e básicas. Possui ampla utilização como sensor resistivo para gases por possuir defeitos na estrutura cristalina devido à variação na valência do estanho entre $\mathrm{Sn}^{2+}$ e $\mathrm{Sn}^{4+}$. Essa variação promove a não estequiometria e cria imperfeições na rede do $\mathrm{SnO}_{2}$, interessantes em aplicações na área tecnológica [5, 6]. Adnan et al. [7] afirmam que a importância do material se dá devido à alta interação química com espécies adsorvidas na superfície do óxido e baixa temperatura de operação.

Desde Komarneni et al. [8], pioneiros na utilização do método hidrotermal assistido por micro-ondas (MHAM) para síntese de pós cerâmicos, a utilização dessa técnica tem se intensificado. Strauss e Rooney [9] recomendam a utilização do MHAM, afirmando que o mesmo tem vantagem sobre o aquecimento convencional no que diz respeito ao tempo. Além disso, é ambientalmente limpo e leva a maiores rendimentos. Essa abordagem ambiental do MHAM tem despertado a atenção na síntese de catalisadores heterogêneos para a produção de biodiesel. O estudo de matérias-primas alternativas na produção de biodiesel, como óleo de fritura, tem se intensificado, porém sua utilização requer pré-tratamento para remoção de água, resíduos sólidos e diminuição do teor de ácidos graxos livres (AGL) [10]. A diminuição dos AGLs se dá por meio da reação de esterificação, transformando-os em ésteres, passíveis de serem aplicados na reação de transesterificação.

A utilização do $\mathrm{SnO}_{2}$ como catalisador para obtenção de biodiesel foi investigada por Santos et al. [11] cuja conversão 
catalítica do óleo vegetal obteve um rendimento de $90 \%$. Srilatha et al. [12] utilizaram o dióxido de estanho como suporte catalítico do ácido tungfosfórico na esterificação metanólica de ácidos graxos. A utilização de $15 \%$ do catalisador levou à conversão de $81,2 \%$ dos ácidos graxos em biodiesel. Este trabalho teve como objetivo sintetizar o $\mathrm{SnO}_{2}$ dopado com nióbio pelo método hidrotermal assistido por micro-ondas e verificar sua aplicação na esterificação do ácido oleico.

\section{MATERIAIS E MÉTODOS}

\section{Síntese dos pós}

A síntese do óxido de estanho foi adaptada da metodologia proposta por Santos [13]. A 22,15 $\mathrm{mmol}$ de cloreto de estanho II dihidratado $\left(\mathrm{SnCl}_{2} \cdot 2 \mathrm{H}_{2} \mathrm{O}\right)$ foi adicionado ácido nítrico $\left(\mathrm{HNO}_{3}\right.$, P.A.) a fim de oxidar o estanho (II) a estanho (IV), de acordo com a Equação A:

$$
\mathrm{Sn}_{(\mathrm{aq})}^{2+}+2 \mathrm{HNO}_{3(\mathrm{aq})} \rightarrow \mathrm{Sn}_{(\mathrm{aq})}^{4+}+\mathrm{H}_{2} \mathrm{O}_{(\mathrm{l})}+\mathrm{NO}_{2(\mathrm{~g})}+\mathrm{NO}_{(\mathrm{g})}+\mathrm{O}_{2(\mathrm{~g})}
$$

Após a eliminação de todo o $\mathrm{NO}_{\mathrm{x}}$, foram adicionados 70 $\mathrm{mL}$ de água destilada. Em seguida adicionou-se o hidróxido de amônio $\left(\mathrm{NH}_{4} \mathrm{OH}, \mathrm{P} . \mathrm{A}\right.$.) até pH 2. Para a síntese dos pós com razão 10:1 ( $\mathrm{Sn}: \mathrm{Nb})$ foram utilizados dois precursores do nióbio, o etóxido de nióbio $\left[\mathrm{Nb}\left(\mathrm{OCH}_{2} \mathrm{CH}_{3}\right)_{5}\right]$ e o pentóxido de nióbio $\left(\mathrm{Nb}_{2} \mathrm{O}_{5}\right)$. Na reação com óxido de nióbio, após eliminação do $\mathrm{NO}_{\mathrm{x}}$, foram adicionadas à mistura gotas de HF P.A. sob agitação constante, a fim de dissociar o $\mathrm{Nb}^{5+}$ do $\mathrm{Nb}_{2} \mathrm{O}_{5}$. Após essa etapa foi feita a lavagem do material obtido, para eliminação do HF.

$\mathrm{Na}$ síntese hidrotérmica por micro-ondas, foi utilizado um forno convencional modificado para sínteses de nanomateriais, com frequência de $2,45 \mathrm{GHz}$ e potência de $800 \mathrm{~W}$. Colocouse a mistura em um copo de teflon com capacidade máxima de $140 \mathrm{~mL}$ e, então, acoplou-se ao reator hidrotérmico nas condições de $2,98 \mathrm{~atm}, 140{ }^{\circ} \mathrm{C}$, tempo reacional de $60 \mathrm{~min}$ e taxa de aquecimento de $10^{\circ} \mathrm{C} \cdot \mathrm{min}^{-1}$. Por fim, a solução foi retirada do reator, passando por um processo de lavagem do precipitado, a fim de retirar o excesso de cloreto. Após a lavagem, o pó foi levado a uma estufa para a secagem a $80^{\circ} \mathrm{C}$ por $12 \mathrm{~h}$. Os pós obtidos da síntese do óxido de estanho sem modificação e modificados pelos precursores pentóxido de nióbio e etóxido de nióbio foram nomeados de S100, SNF e SNL, respectivamente.

\section{Caracterização}

A análise de microscopia foi realizada por intermédio de um microscópio eletrônico de varredura com canhão de elétrons por emissão de campo (MEV-FEG, Supra 35-VP, Carl Zeiss, Alemanha) operado com um feixe de elétrons incidentes de $6 \mathrm{kV}$. O preparo das amostras começou com a dispersão de aproximadamente $1 \mathrm{mg}$ de cada pó em $20 \mathrm{~mL}$ de acetona. Em seguida, cada suspensão foi submetida a $10 \mathrm{~min}$ de ultrassom para dispersar as partículas. Na sequência, uma alíquota desta suspensão foi depositada sobre um substrato de silício, este aderido a um porta-amostra de alumínio por fita de carbono. Após a evaporação do solvente, foi utilizada tinta prata, com intuito de fazer o contato elétrico entre a superfície para análise e o porta-amostra. A análise elementar foi obtida por meio de um microscópio Phenon Pro X, equipado com um espectrômetro de energia dispersiva de raios $\mathrm{X}$ (EDS).

A estrutura cristalina e a formação da fase foram verificadas por meio da difração de raios $\mathrm{X}$, utilizando um difratômetro PANalytical, X'Pert Pro (Mpdpw 3040/60), usando radiação $\mathrm{CuK} \alpha(\lambda=1,5418 \AA)$, com intervalo de

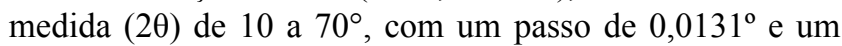
tempo de contagem de 7,564 s. Para a identificação das fases cristalinas utilizou-se o software HighScore Plus v. 3.0 e a base de dados PDF 2-ICDD v. 2.4. Os tamanhos de cristalito foram calculados utilizando a equação de Scherrer, dada por:

$$
\mathrm{D}=\frac{\mathrm{K} . \lambda}{\beta \cos \theta}
$$

em que, $D$ é o diâmetro médio das partículas, $K$ representa a constante dependente da forma das partículas, $\lambda$ é o comprimento de onda, $\beta$ é a largura à meia altura do pico mais intenso e $\theta$ o ângulo de difração. Foram consideradas as intensidades do pico principal (110) e o cálculo foi realizado considerando partículas esféricas $(\mathrm{K}=0,9)$. As análises de espectroscopia de infravermelho com transformada de Fourier (FTIR) foram realizadas em um espectrofotômetro IRPrestige-21 da Shimadzu, dispersando o material em pastilhas de brometo de potássio $(\mathrm{KBr})$, com leitura realizada na faixa de 4000 a $400 \mathrm{~cm}^{-1}$ e resolução de $4 \mathrm{~cm}^{-1}$.

\section{Aplicação catalítica}

Os testes catalíticos foram realizados em um reator da Parr, série 4560, com um controlador modelo 4848. As reações de esterificação ocorreram numa razão molar 1:20 (ácido oleico : álcool metílico), utilizando $1 \mathrm{~g}$ de catalisador a $100{ }^{\circ} \mathrm{C}$ por $4 \mathrm{~h}$. O catalisador foi ativado a $60{ }^{\circ} \mathrm{C}$ por 1 $\mathrm{h}$ a fim de proporcionar um aumento na energia cinética média das moléculas e com isso obter maior probabilidade destas moléculas atingirem uma energia igual à energia de ativação da reação, com intuito de produzir uma maior quantidade de produtos em menor tempo. O oleato de metila obtido foi caracterizado por FTIR, viscosidade cinemática e cromatografia a gás acoplada a espectrometria de massas. Para as medidas de viscosidade, foi utilizado um tubo capilar Cannon-Fenske $\mathrm{n}^{\circ} 75$, em um banho termostático Julabo a $40{ }^{\circ} \mathrm{C}$. O cálculo da viscosidade foi obtido por:

$$
v=C . t
$$

em que, $v$ representa a viscosidade cinemática $\left(\mathrm{mm}^{2} \cdot \mathrm{s}^{-1}\right)$, $C$ é a constante do viscosímetro $\left(\mathrm{mm}^{2} \cdot \mathrm{s}^{-2}\right)$, que depende do diâmetro, da inclinação e do volume escoado no capilar, e $t$ é o tempo de escoamento da amostra (s). A dimensão 
da constante é $\mathrm{C}=\mathrm{L}^{2} / \mathrm{t}^{2}$, em que $\mathrm{L}$ é o comprimento do tubo $(\mathrm{mm})$. As amostras permaneceram por $20 \mathrm{~min}$ na temperatura de $40{ }^{\circ} \mathrm{C}$ e, então, iniciaram-se as análises. Depois de marcado o tempo de escoamento, multiplicouse o mesmo pela constante do capilar $\left(0,008064 \mathrm{~mm}^{2} . \mathrm{s}^{-2}\right) \mathrm{e}$ obteve-se a viscosidade. Para esta análise, as medidas foram realizadas em triplicata.

\section{Determinação analítica}

As análises foram realizadas em um cromatógrafo a gás (GC 2010), acoplado a um espectrômetro de massas (MS), GCMS-QP2010Plus, ambos da Shimadzu, utilizando a coluna capilar SLB ${ }^{\mathrm{TM}}-5 \mathrm{~ms}(60 \mathrm{~m} \times 0,25 \mathrm{~mm}$ de diâmetro interno). $\mathrm{O}$ gás de arraste utilizado foi o hélio em um fluxo de $1 \mathrm{~mL} \cdot \mathrm{min}^{-1}$ e razão de split de 1:100. Foi injetado $1 \mu \mathrm{L}$ da amostra diluída em acetonitrila. As temperaturas do injetor e da interface foram estabelecidas, ambas, em $250{ }^{\circ} \mathrm{C}$ e a programação de temperatura do forno de 130 a $220^{\circ} \mathrm{C}$ com isotermas em 223 e $250{ }^{\circ} \mathrm{C}$ e taxas de aquecimento de 20 e $6{ }^{\circ} \mathrm{C} \cdot \mathrm{min}^{-1}$. Com relação ao MS, os dados de aquisição foram no modo scan $0,30 \mathrm{~s}^{-1}$ em um faixa de 35 a $500 \mathrm{~m} / \mathrm{z}$. Os compostos foram identificados através dos espectros de massas, de acordo com a biblioteca do equipamento, National Institute of Standards and Tecnology (NIST 0.8). Os valores de conversão em oleato de metila foram obtidos pelo método de normalização de área (\% em área) $[14,15]$, realizada pelo software por meio da equação:

$$
\%_{\mathrm{i}}=\mathrm{A}_{\mathrm{i}} / \sum \mathrm{A}_{\mathrm{i}} \cdot 100
$$

em que, $\mathrm{A}_{\mathrm{i}}$ é a área do composto $i$ e $\sum \mathrm{A}_{\mathrm{i}}$ é a somatória das áreas de todos os compostos.

\section{RESULTADOS E DISCUSSÃO}

Os espectros de FTIR da Fig. 1 apresentam os principais modos vibracionais presentes nos catalisadores S100, SNF e SNL. As bandas de absorção entre 3500 e $3000 \mathrm{~cm}^{-1}$ e em $1634 \mathrm{~cm}^{-1}$ são atribuídas à presença de água na superfície da amostra e referem-se aos estiramentos das ligações $\mathrm{O}-\mathrm{H}$ e $\mathrm{H}-\mathrm{O}-\mathrm{H}$, respectivamente. As bandas em 552, 676 e $1381 \mathrm{~cm}^{-1}$ são atribuídas às vibrações das ligações estanhooxigênio, referentes às ligações $\mathrm{Sn}-\mathrm{O}, \mathrm{O}-\mathrm{Sn}-\mathrm{O}$ e Sn-O$\mathrm{Sn}$, respectivamente [16]. O aparecimento da banda na região de 500 a $600 \mathrm{~cm}^{-1}$ pode ser atribuído ao estiramento da ligação $\mathrm{Nb}-\mathrm{O}$ [17]. No espectro do $\mathrm{SNL}$, notam-se ainda modos vibracionais atribuídos à ligação $\mathrm{C}-\mathrm{H}$. Estes modos podem estar relacionados à presença de etóxido de nióbio $\left[\mathrm{Nb}\left(\mathrm{OCH}_{2} \mathrm{CH}_{3}\right)_{5}\right]$ residual na amostra. Em 1398 $\mathrm{cm}^{-1}$ observa-se uma banda, presente nas três amostras, que indica a presença de carbonato adsorvido na superfície das mesmas [18].

Na Fig. 2 são mostrados os difratogramas de raios $\mathrm{X}$ para as amostras sintetizadas. Segundo a análise dos difratogramas, os pós apresentaram o padrão de difração característico do óxido de estanho IV $\left(\mathrm{SnO}_{2}\right)$, de acordo

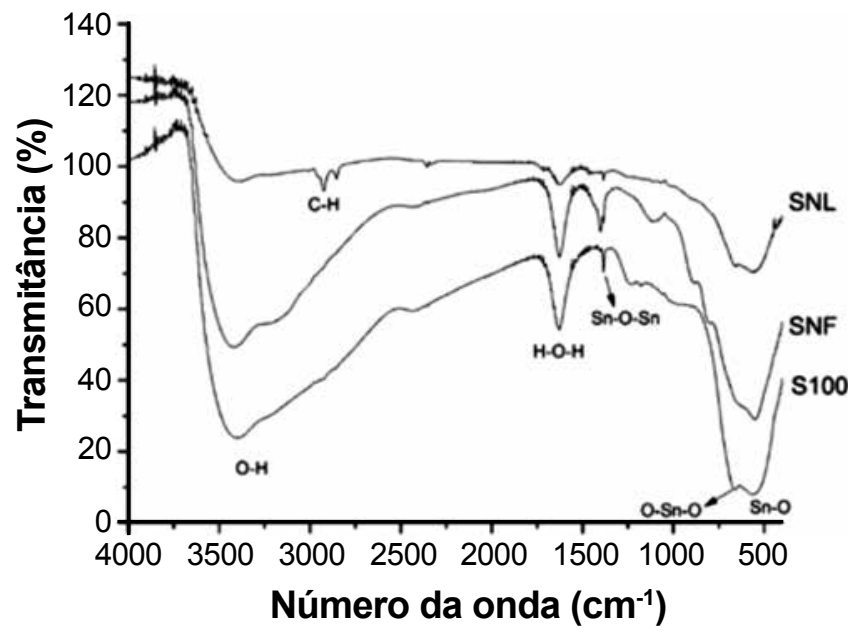

Figura 1: Espectros de infravermelho dos catalisadores S100, SNL e SNF.

[Figure 1: FTIR spectra of S100, CNS and SNF catalysts.]

com o arquivo JCPDS 41-1445. As reflexões em $2 \theta=$ $26^{\circ}, 33^{\circ}, 37^{\circ}, 51^{\circ}$ e $64^{\circ}$ referem-se aos planos (110), (101), (200), (211) e (002), respectivamente, e comprovam que a estrutura rutilo foi obtida. A diferença entre os materiais de partida dos dopantes não causou mudanças evidentes na análise de DRX, pois os difratogramas apresentaram perfis semelhantes, ocorrendo apenas a diminuição da intensidade da reflexão referente ao plano (101) para o SNF.

Os tamanhos de cristalito foram calculados utilizando a equação de Scherrer (Eq. B). Os dados da Tabela I indicam a tendência da formação de material nanométrico, como previsto pelo perfil dos difratogramas das amostras, exceto para o SNF que apresentou picos mais alargados, porém maior tamanho de cristalito. Segundo Sousa [19], quanto menor o cristalito, maior é a deformação da célula unitária, que pode tornar o pico mais alargado. Partículas nanométricas

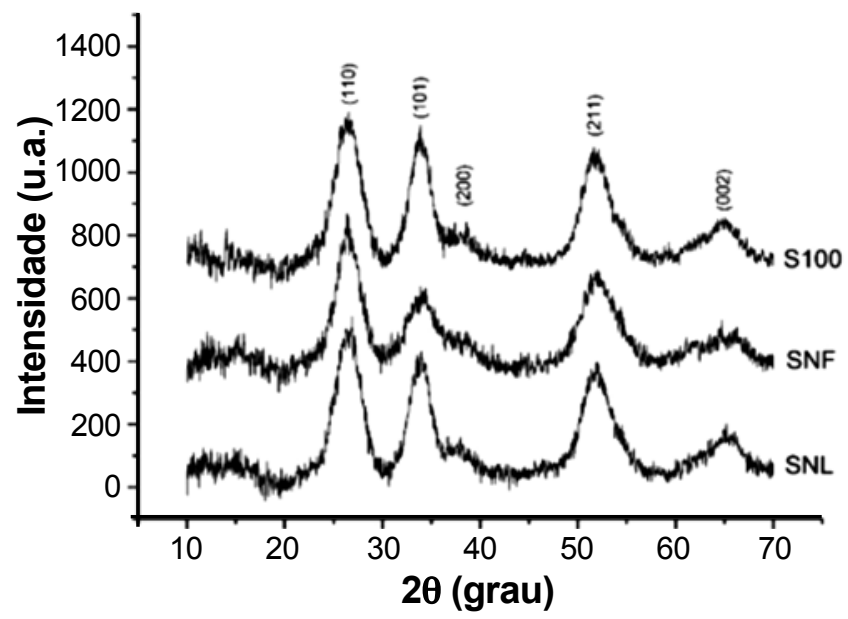

Figura 2: Difratogramas de raios X dos catalisadores S100, SNF e SNL sintetizados em $\mathrm{pH} 2$.

[Figure 2: X-ray diffraction patterns of S100, SNH and SNL catalysts synthesized at $\mathrm{pH}$ 2.] 
Tabela I - Tamanhos médios de cristalito dos catalisadores sintetizados.

[Table I - Average crystallite sizes of synthesized catalysts.]

\begin{tabular}{cc}
\hline Catalisador & Tamanho de cristalito $(\mathrm{nm})$ \\
\hline S100 & 5,5 \\
SNL & 5,2 \\
SNF & 6,3 \\
\hline
\end{tabular}
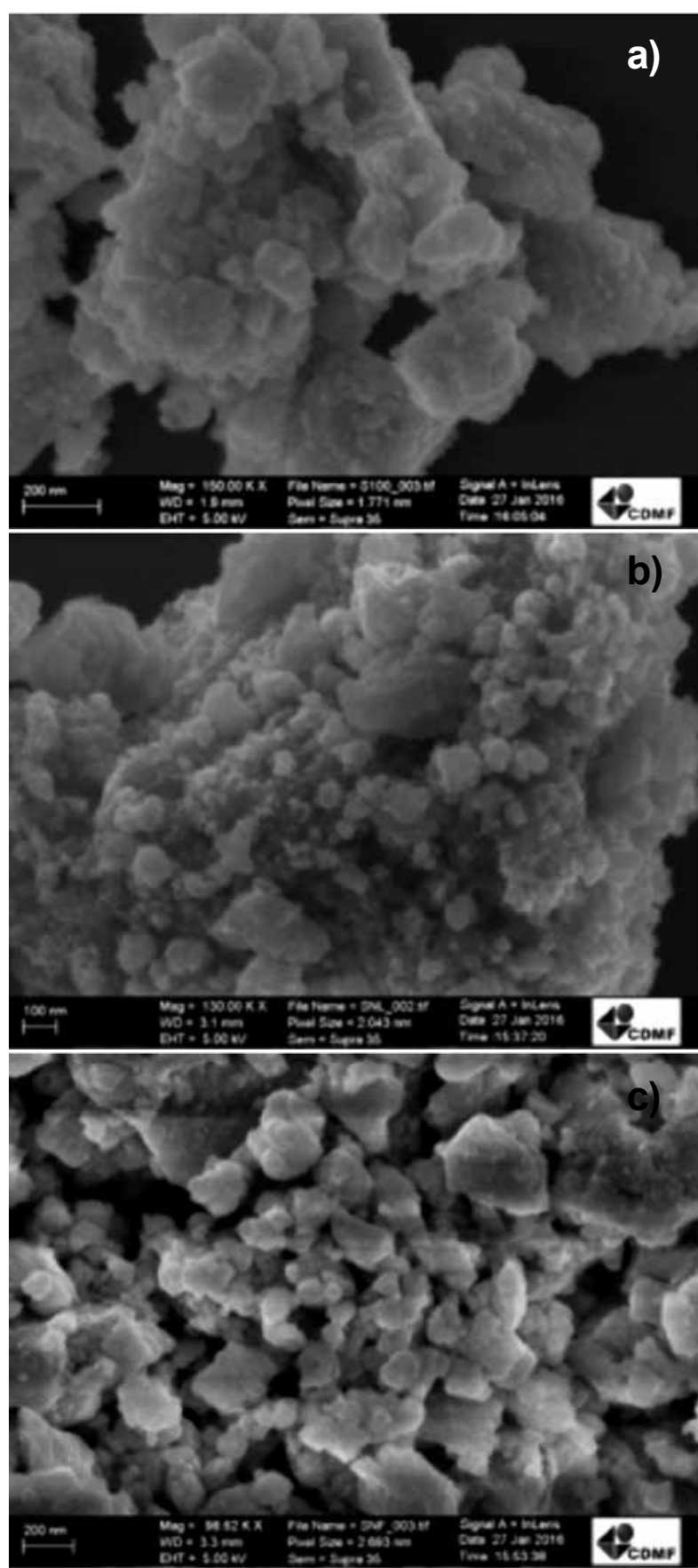

Figura 3: Micrografias obtidas por microscopia eletrônica de varredura MEV-FEG mostrando a formação de aglomerados nos sistemas (a) SN100, (b) SNL e (c) SNF.

[Figure 3: SEM-FEG micrographs showing the formation of agglomerates in the systems (a) SN100, (b) SNL and (c) SNF.] tendem a formar aglomerados com tendência à morfologia esférica, diminuindo assim a energia superficial [20], o que pode ter ocorrido para o sistema SNF. As micrografias obtidas por MEV-FEG, Fig. 3, mostram a morfologia das partículas obtidas, em que se podem observar aglomerados de partículas nanométricas para os três sistemas sintetizados.

A análise de EDS, Fig. 4, mostra a distribuição do nióbio para o catalisador SNL, bem como as proporções desse dopante nos pontos analisados (Tabela II). Para o sistema SNF a distribuição do dopante se mostrou mais uniforme (Tabela III). A melhor distribuição do dopante pode melhorar a atividade catalítica do SNF em comparação ao SNL, visto que os sítios ativos promovem maior superfície de contato com os reagentes.

Após a caracterização dos pós S100, SNL e SNF, realizou-se o teste catalítico na reação de esterificação do ácido oleico. A Fig. 5 mostra o espectro comparativo de modos vibracionais característicos dos catalisadores e do oleato de metila resultante da reação de esterificação do ácido oleico. As principais bandas características estão localizadas em 1170, 1022 e $960 \mathrm{~cm}^{-1}$ que representam as deformações axiais dos grupos $\mathrm{C}-\mathrm{C}(\mathrm{C}=\mathrm{O})-\mathrm{O}$ e $\mathrm{O}-\mathrm{C}-\mathrm{C}$ [21]. Observa-se ainda o aparecimento da banda em 1740 $\mathrm{cm}^{-1}$, região característica de carbonila, porém para os produtos das reações ocorre em maior intensidade devido à contribuição do grupo metil acoplado.

As medidas de viscosidade cinemática são mostradas na Fig. 6. De acordo com a técnica, a maior conversão em ésteres pode ser caracterizada pela diminuição da viscosidade. A viscosidade do oleato de metila a $40{ }^{\circ} \mathrm{C}$ é $4,3 \mathrm{~mm}^{2} . \mathrm{s}^{-1}$ e o sistema catalisador sintetizado que mais se aproximou desse valor foi o S100, dióxido de estanho puro com viscosidade de $7,18 \mathrm{~mm}^{2} . \mathrm{s}^{-1}$. Essa redução acontece devido à substituição da hidroxila no grupo carbonila pelo grupo alquil do éster. A diminuição nesse grupo faz com que existam menos interações entre eles através das ligações de hidrogênio, reduzindo assim a viscosidade.

Os cromatogramas do ácido oleico e das misturas reacionais estão relacionados na Fig. 7. Nos cromatogramas $\mathrm{B}, \mathrm{C}$ e $\mathrm{D}$, que correspondem aos produtos de síntese com os catalisadores S100, SNL e SNF, respectivamente, observase uma diminuição na intensidade do pico que corresponde ao ácido oleico (18,2 $\mathrm{min})$; porém, é possível constatar também a não conversão total do ácido oleico em oleato de metila $(17,4 \mathrm{~min})$, uma vez que o mesmo está presente nos respectivos cromatogramas, sendo confirmado com valores obtidos pelo método de normalização, onde se tem $36,9,35,7$ e 41,6\% para os catalisadores S100, SNL e SNF, respectivamente. Os valores divergiram dos resultados da viscosidade cinemática, ou seja, esperar-se-ia que o sistema S100 apresentasse um maior rendimento devido à menor viscosidade obtida. Porém, a melhor conversão obtida foi para o sistema SNF. Tal resultado corrobora com a morfologia das amostras obtidas por MEV, uma vez que o sistema SNF apresentou menos aglomerados que nos demais sistemas, permitido uma maior interação entre os reagentes e o catalizador. Também se verificou que neste sistema a 


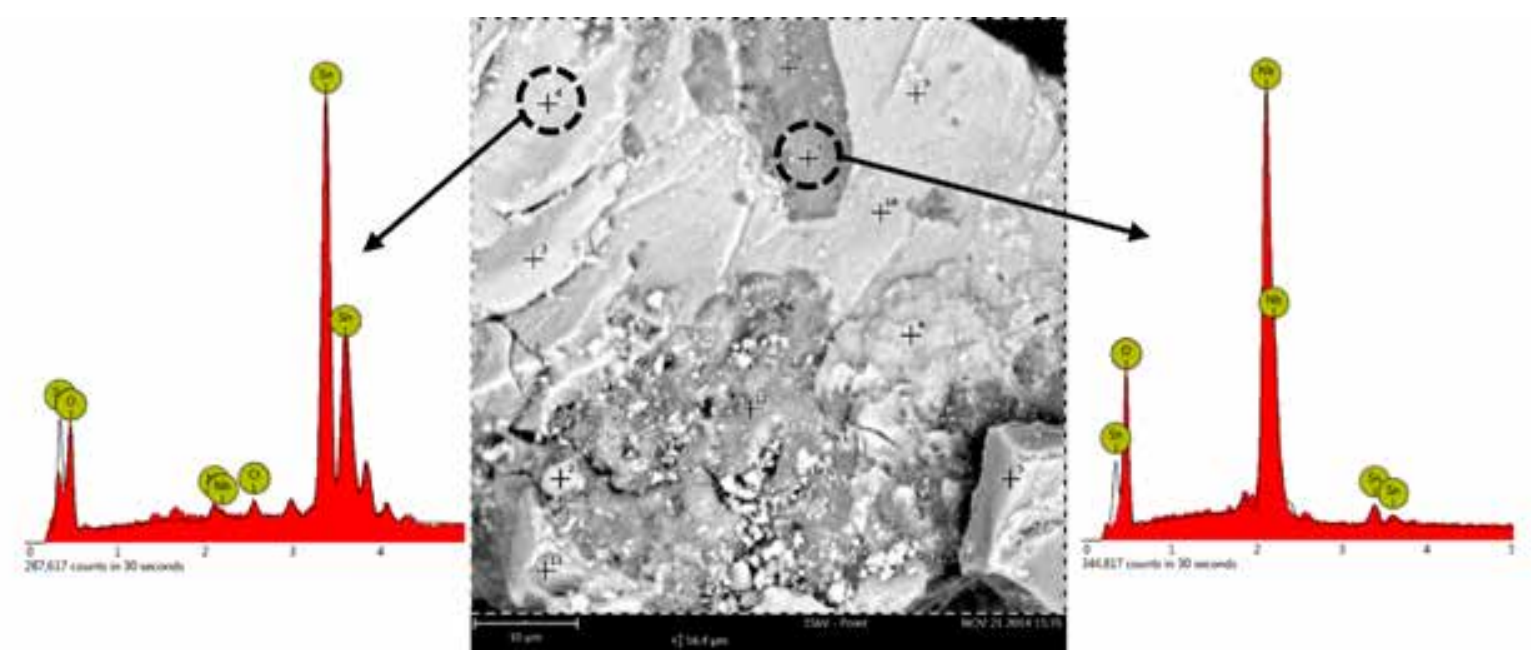

Figura 4: Micrografia obtida por microscopia eletrônica de varredura e espectros de EDS do sistema SNL. [Figure 4: SEM micrograph and EDS spectra of the SNL system.]

Tabela II - Resultados da análise de EDS do sistema SNL. [Table II - Results of EDS analysis of the SNL system.]

\begin{tabular}{ccc}
\hline Região da amostra & $\mathrm{Sn}(\% \mathrm{em} \mathrm{mol})$ & $\mathrm{Nb}(\% \mathrm{em} \mathrm{mol})$ \\
\hline 1 & 10,2 & 6,2 \\
2 & 24,1 & 75,9 \\
3 & 14,5 & 0,3 \\
4 & 19,0 & 0,6 \\
5 & 15,1 & 0 \\
6 & 11,0 & 3,1 \\
7 & 1,2 & 15,0 \\
8 & 1,1 & 14,9 \\
9 & 12,9 & 0 \\
10 & 12,6 & 0 \\
11 & 37,3 & 1,9 \\
12 & 1,8 & 16,1 \\
\hline
\end{tabular}

Tabela III - Resultados da análise de EDS do sistema SNF. [Table III - Results of EDS analysis of the SNF system.]

\begin{tabular}{ccc}
\hline Região da amostra & $\mathrm{Sn}(\% \mathrm{em} \mathrm{mol})$ & $\mathrm{Nb}(\% \mathrm{em} \mathrm{mol})$ \\
\hline 1 & 75,6 & 14,2 \\
2 & 79,3 & 10,5 \\
3 & 75,4 & 13,5 \\
4 & 25,6 & 5,3 \\
5 & 75,9 & 13,9 \\
6 & 75,3 & 14,9 \\
7 & 75,8 & 4,6 \\
8 & 78,0 & 9,3 \\
9 & 75,3 & 14,0 \\
\hline
\end{tabular}

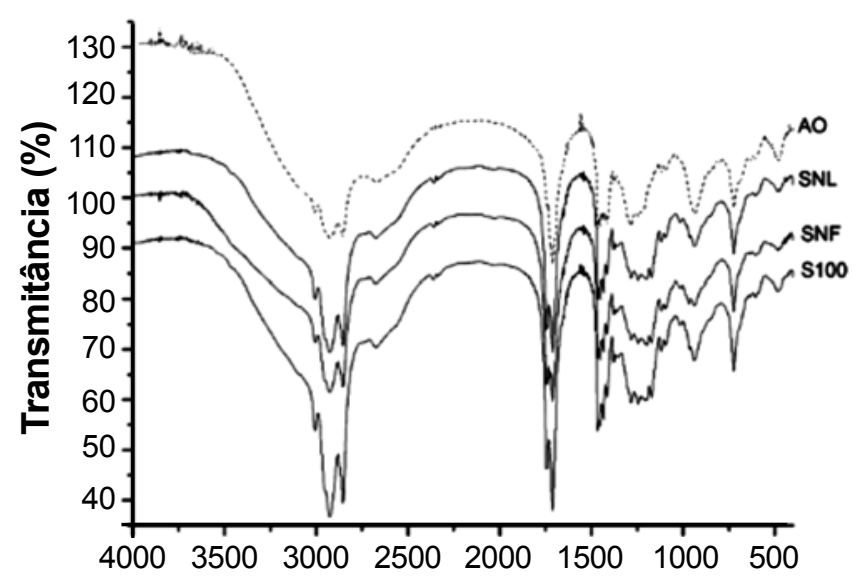

\section{Comprimento da onda $\left(\mathrm{cm}^{-1}\right)$}

Figura 5: Espectros de infravermelho dos produtos das reações e ácido oleico.

[Figure 5: FTIR spectra of the products of reactions and oleic acid.]

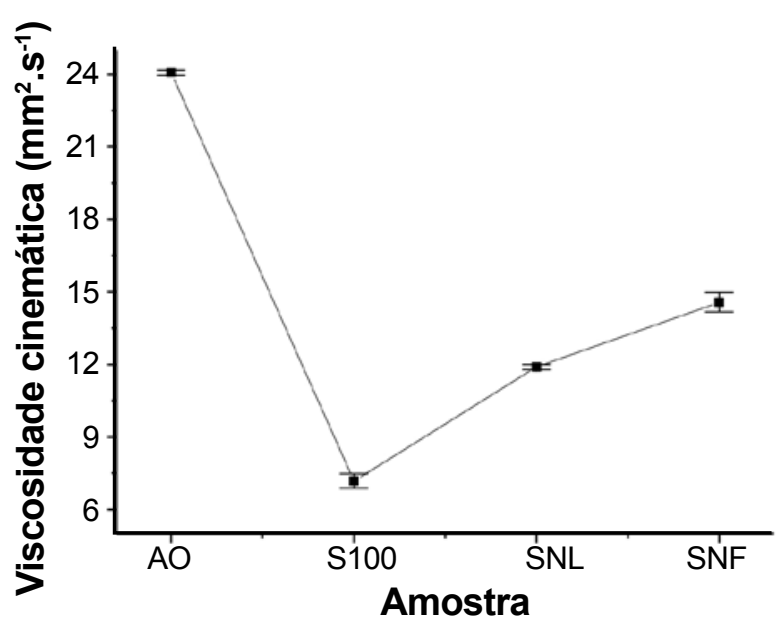

Figura 6: Viscosidade cinemática dos produtos da reação de esterificação e ácido oleico (AO).

[Figure 6: Kinematic viscosity of the products of esterification reaction and oleic acid $(A O)$.] 

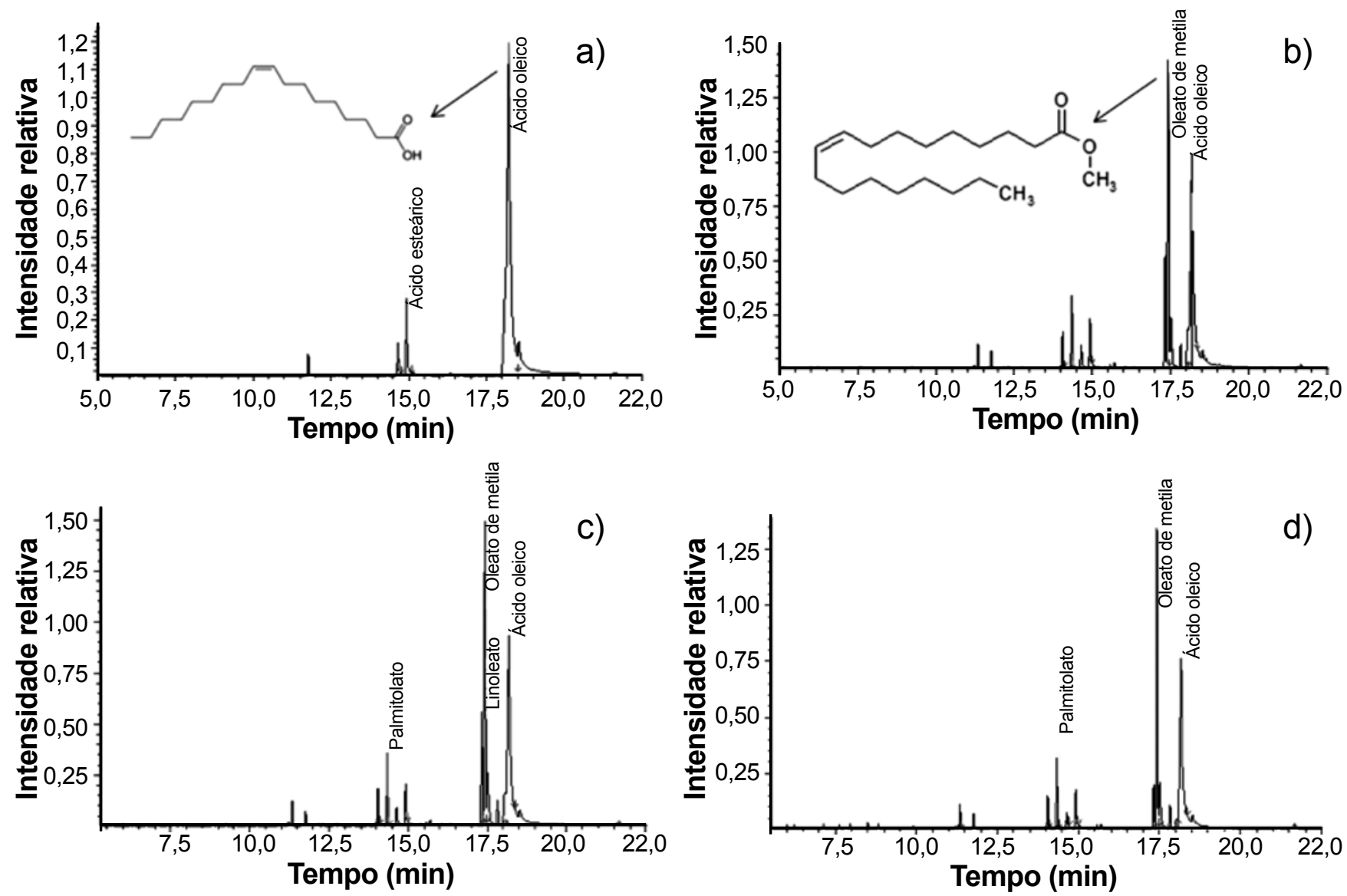

Figura 7: Cromatogramas do reagente de partida, ácido oleico (A), e dos produtos de síntese com os catalisadores S100 (B), SNL (C) e SNF (D). [Figure 7: Chromatograms of the reagent, oleic acid (A), and products of synthesis with the catalysts $S 100(B), S N L(C)$ and $S N F(D)$.]

distribuição do nióbio é mais uniforme, promovendo assim uma maior conversão do oleato de metila.

\section{CONCLUSÕES}

O óxido de estanho (IV) modificado com nióbio foi sintetizado pelo método hidrotérmico assistido por microondas. A fase cassiterita foi confirmada pela análise por difração de raios $\mathrm{X}$, bem como os modos vibracionais referentes à ligação metal oxigênio dos sistemas sintetizados analisados por FTIR. A tendência de partículas nanométricas foi corroborada pelo tamanho médio de cristalitos obtidos e os padrões de difração de raios $\mathrm{X}$ dos materiais, bem como a análise morfológica obtida por MEV-FEG. A análise por EDS mostrou uma distribuição do $\mathrm{Nb}$ mais uniforme no sistema SNF, que no sistema SNL, o que pode ter contribuído para uma atividade mais efetiva deste catalisador com conversão de $41,6 \%$ em oleato de metila.

\section{AGRADECIMENTOS}

A CAPES e FAPEMA pelo aporte financeiro, ao LIECUFSCar e à prof. Içamira Costa Nogueira pela realização de análises de MEV-FEG e ao Núcleo de Catálise, Combustíveis e Ambiental da UFMA.

\section{REFERÊNCIAS}

[1] F. Galembeck, M. do C.V.M. Silva, R. Rosseto, Quim. Nova 30, 3 (2007) 745.

[2] X.W. Lou, Y. Wang, C. Yuan, J.Y. Lee, L.A. Archer, Adv. Mater. 18, 17 (2006) 2325.

[3] N.L. Rosy, C.A. Mirkin, Chem. Rev. 105, 4 (2005) 1547. [4] D. Li, Q. Li, X. Hao, Y. Zhang, Z. Zhang, C. Li, Curr. Top. Med. Chem. 14, 5 (2014) 595.

[5] A.P. Maciel, E. Longo, E.R. Leite, Quim. Nova 26, 6 (2003) 855.

[6] P.D. Borges, "Propriedades físicas do $\mathrm{SnO}_{2}$ : defeitos, impurezas, ligas e super-redes", Tese Dr., Instituto de Física da Universidade de S. Paulo, SP (2011).

[7] R. Adnan, N.A. Razana, I.A. Rahman, M.A. Farrukh, J. Chin. Chem. Soc. 57 (2010) 222.

[8] S. Komarneni, R. Roy, Q. H. LI, Mat. Res. Bull. 27 (1992) 1393.

[9] C.S. Strauss, D.W. Rooney, Green Chem. 12 (2010) 1340.

[10] Z. Yaakob, M. Mohammad, M. Alherbawi, Z. Alam, K. Sopian, Renew. Sust. Energ. Rev. 18 (2013) 184.

[11] A.M. dos Santos, J.S. Vasconcelos, J.H.G. Rangel, E. Longo, M.M. Oliveira, Cerâmica 60 (2014) 397.

[12] K. Srilatha, Ch. RameshKumar, B.L.A. Prabhavathi 
Devi, R.B.N. Prasad, P.S. Sai Prasad, N. Lingaiah, Catal. Sci. Technol. 1 (2011) 662.

[13] A.M. Santos, "Síntese de nanocatalisadores cerâmicos a base de $\mathrm{SnO}_{2}$ dopado com Mo e Pt pelo método hidrotermal assistido por micro-ondas", Diss. Mestrado, Instituto Federal de Educação, Ciência e Tecnologia do Maranhão, MA (2013).

[14] E. Engel, J. Ratel, J. Chomatography A 1154 (2007) 331.

[15] M. Chen, R.S.P. Rao, Y. Zhang, C.X. Zhong, J. Thelen, Springer Plus 3 (2014) 439.

[16] L. Tan, L. Wang, Y. Wang, J. Nanomater. 2011 (2011) 1.

[17] D. Thomazini, F. Lanciotti Jr., A.S.B. Sombra, Cerâmica 47, 302 (2001) 88.
[18] D. Amalric-Popescu, F. Bozon-Verduraz, Catal. Today 70 (2001) 139.

[19] D.G. Sousa, "Síntese de nanopartículas de $\mathrm{SnO}_{2}$ dopadas com Ni pelo método hidrotermal assistido por micro-ondas para aplicação como catalisador heterogêneo na reação de transesterificação para obtenção de biodiesel", Diss. Mestrado, Instituto Federal de Educação, Ciência e Tecnologia do Maranhão, MA (2012).

[20] P.G. Mendes, M.L. Moreira, S.M. Tebcherani, M.O. Orlandi, J. Andrés, M.S. Li, N.D. Mora, J.A. Varela, E. Longo, J. Nanopart. Res. 14 (2012) 1.

[21] B. Meesaragandla, V.N. Adusumalli, V. Mahalingam, Langmuir 31, 19 (2015) 5521.

(Rec. 02/02/2016, Rev. 21/03/2016, Ac. 06/04/2016) 